\title{
Computer-Aided Prototype System for Nose Surgery
}

\author{
Tong-Yee Lee, Chao-Hung Lin, and Han-Ying Lin
}

\begin{abstract}
Rhinoplasty, or surgery to reshape the nose, is one of the most common of all plastic-surgery procedures. Rhinoplasty can enhance a patient's appearance and self-confidence, may also correct a birth defect or injury, or help relieve some breathing problem. In this paper, we present a three-dimensional (3-D) surgical simulation system, which can assist surgeons in planning rhinoplasty procedures. This system employs computer graphics and image-processing techniques for the simulation of a rhinoplasty. Although the presented algorithms themselves are not new, the proposed system exploits the new idea to apply 3-D morphing for rhinoplasty, and simulation results are useful for the physicians. According to patients' expectation of what they would like their noses to look like, our system simulates expected results. Our tools provide quantitative measurements of a nose structure. Using these quantitative results, surgeons can arrange appropriate preoperative plans for patients. Finally, experimental results and experiences are reported to evaluate the usefulness of the proposed system.
\end{abstract}

Index Terms-Morphing operator, rhinoplasty, surgical simulation, volume morphing.

\section{INTRODUCTION}

$\mathbf{T}$ HERE ARE many reasons why people would like to have nose surgery. Some of these reasons include dissatisfaction with the appearance of their noses or the effects of aging. Rhinoplasty, or surgery to reshape the nose, is one of the most common of all plastic-surgery procedures. Rhinoplasty can reduce or increase the size of the nose, change the shape of the tip or the bridge, narrow the span of nostrils, or change the angle between the nose and upper lip. Additionally, it may also correct a birth defect or injury, or help relieve some breathing problems.

Before rhinoplasty, patients must think carefully about their expectations and discuss them with the surgeons. At this stage, good communication between patients and physicians is essential. For example, the patients always prefer that the nasal dorsum should be smooth and the nose tip should be moderately sharp. However, the beauty of a nose is also dependent on other parts of the patient such as the forehead, eyes, and chin [1]. Therefore, besides the expectation of patients, surgeons need to evaluate the structure of the nose and face and then make an appropriate surgical planning.

Each operation is planned individually with feedback from the patients in order to realize a nose with shape comfortable to the patients. In this paper, we present a three-dimensional

Manuscript received May 31, 2000; revised January 11, 2001. This work was supported by the National Science Council, R.O.C. under Grant NSC 89-2218E006-028.

The authors are with the Computer Graphics Group, Visual System Laboratory, Department of Computer Science and Information Engineering, National Cheng-Kung University, Tainan, Taiwan, R.O.C. (e-mail: tonylee@mail.ncku.edu.tw).

Publisher Item Identifier S 1089-7771(01)07413-1.
(3-D) surgical simulation system for nose surgeries. With this system, surgeons can simulate surgery to reshape the nose, and quantitatively characterize the nose structure such as the nasal tip.

In the past, there have been several related approaches proposed [1]-[4]. Achermann et al. [1] presented a tool that can assist surgeons in judging on the success of nose surgeries. The tool described in [1] was based on several lateral photographs [two-dimensional (2-D)], i.e., pictures of the profile of the patients. Our proposed system makes use of similar methods for the quantitative measurement of human noses. However, all measurements were obtained in 3-D and potentially provide more useful information to clinicians. Patel et al. [2] defined and tested a methodology for comparing surgical simulations to postoperative outcomes. This work simulates surgical correction of selected congenital and acquired craniofacial deformities, and applies the comparison criteria to surgical simulations. Linney et al. [5] presented automated tools for landmark determination on the facial profile features, and for the planning and evaluation of facial surgery based on anthropometry. Koch et al. [6] proposed algorithms in both presurgical planning and postsurgical evaluation. In this approach, the facial surgery is realistically simulated using finite-element methods. Several studies such as [7] and [8] concentrated on the development of a more general and flexible muscle model for parameterization that allows facial control to generate realistic facial expression. However, these approaches are always computationally very expensive; they are not practical for real-time or interactive surgical simulation. In forensic medicine, several studies were aimed at reconstructing the face of a deceased person from the skull of a given cadaver [9]-[11]. Our recent study [12] applied both metamorphosis and vision techniques to reconstruct a 3-D facial model from a given model. Additionally, in this study, we also designed several interesting morphing operations allowing realistic plastic-surgery simulation.

This paper is organized as follows. Section II will briefly overview the proposed system. Section III will present the 3-D feature-based volume morphing technique used in this paper. Experimental results and quantitative measurements are described in Section IV. Finally, concluding remarks are given in Section V.

\section{3-D COMPuteR-Aided Nose SuRgical Simulation SYSTEM}

In this system, the main processing components include image processing, 3-D volume rendering and morphing, and quantitative measurement of changes in the human nose. Fig. 1 shows the exact processing scenario in the proposed system. Surgeons follow this scenario to simulate surgeries 


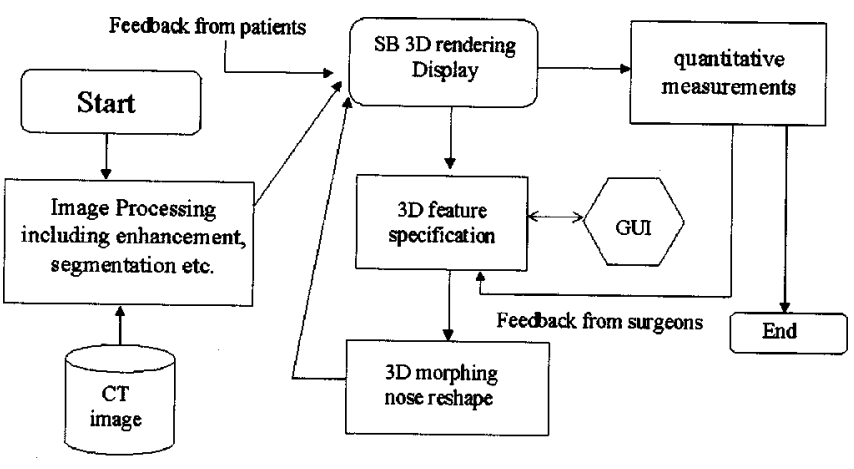

Fig. 1. Processing flowchart of the proposed system.

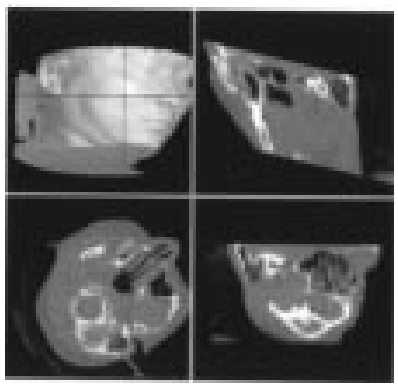

(a)

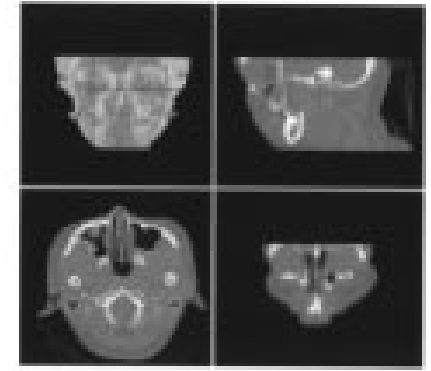

(b)
Fig. 2. 3-D SB surface display system. There are two cross lines (in the right-hand-side top window) used to locate 3-D positions on the object surface. Or simply click mouse buttons on the 3-D surface. (a) Facial structure can be viewed from different viewpoints and three cross-sectional images assist in understanding the spatial relationship of the face. (b) Different segmentation levels can be arbitrarily adjusted to view different structures of the head.

and simultaneously patients feed back their suggestions about what they would like their noses to look like. First, we obtain 3-D computed tomography (CT) head data of a patient. These CT scans were obtained from a GE (high-speed) CT scanner at the National Cheng-Kung University Hospital, Tainan, Taiwan, R.O.C. We then apply image processing to this CT head dataset including image enhancement and segmentation. To interactively display different views of the CT head in 3-D, the CT data is converted to a data structure called semiboundary (SB) [13]. This data structure is a compact voxel surface representation of the structure from the medical images. It represents the boundary of the extracted structure involved in a 3-D volume dataset that can be visualized. Later, Udupa et al. [16] proposed a 3-DVIEWNIX system that was one of the first to demonstrate various surgery simulation and measurement procedures with extremely rapid response time. In this system, the SB method can handle multiple objects that can be rendered in a translucent fashion. In our proposed system, we implement a fast SB rendering method [14] to visualize the CT head. This method achieves an interactive rendering speed for our CT head dataset on PC platforms.

In the proposed system, both the surface of the CT head and three orthogonal views of the volume data are observed at the same time, as shown in Fig. 2(a). Surgeons interactively rotate the CT head to the desired orientations providing more insight for physical examination and findings than using the 2-D image slices. To better understand the head structure, as shown

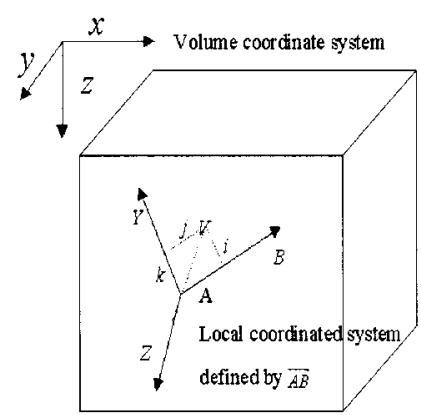

Target Volume

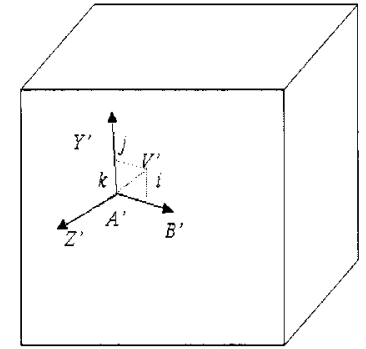

Source volume
Fig. 3. Single feature line pair in volume metamorphosis.

in Fig. 2(b), the CT head can be interactively segmented using different thresholds, and three orthogonal views of the volume data can be viewed slice by slice by clicking the mouse buttons on the 3-D surface image and three orthogonal views. In this figure, we show the bone structure and, therefore, surgeons can examine the bone structure in more detail. This feature is vital to surgical planning, since surgeons must know the 3-D spatial relationship between skin and bone structure to design an appropriate surgical operation.

The surgery to reshape the nose is simulated using a 3-D feature-based morphing technique. This technique allows an interactive simulation of rhinoplasty on PC platforms. In Section III, this morphing technique is described in detail. To apply this technique, we need to specify several feature points in 3-D space on the surface of the CT head. In the proposed system, surgeons first rotate an object to a desired orientation and then simply click the mouse buttons on the 3-D surface to identify the surface position. After simulation, the quantitative measurement for the preoperative and postoperative CT data is performed. This quantitative measurement can assist surgeons in planning the actual nose surgery. In Section IV, we provide additional details about this measurement.

\section{3-D FEATURE-BASED VOLUME MORPHING}

Metamorphosis or warping is a powerful technique to transform one image or a 3-D model into another. Beier and Neely proposed a 2-D feature-based metamorphosis method [15]. This approach gives the animator a high-level control of the visual effect by providing natural feature-based specification and interaction. Using this method, an animator begins with establishing correspondence with pairs of feature line segments on two input images. This method then generates a sequence of intermediate frames between the two input images. In this sequence, the first image is gradually distorted into the second image. In the proposed system, we apply a 3-D extension of Beier et al.'s method to perform 3-D CT volume metamorphosis. Like morphing in 2-D, the proposed system requires surgeons to specify several feature line segments on the 3-D CT head surface. These features are used to control the reshaping of the nose.

In Fig. 3, there are two given volume data of equal size, i.e., target and source, where source is preoperative CT volume data and target is postoperative volume data. Each voxel of the 


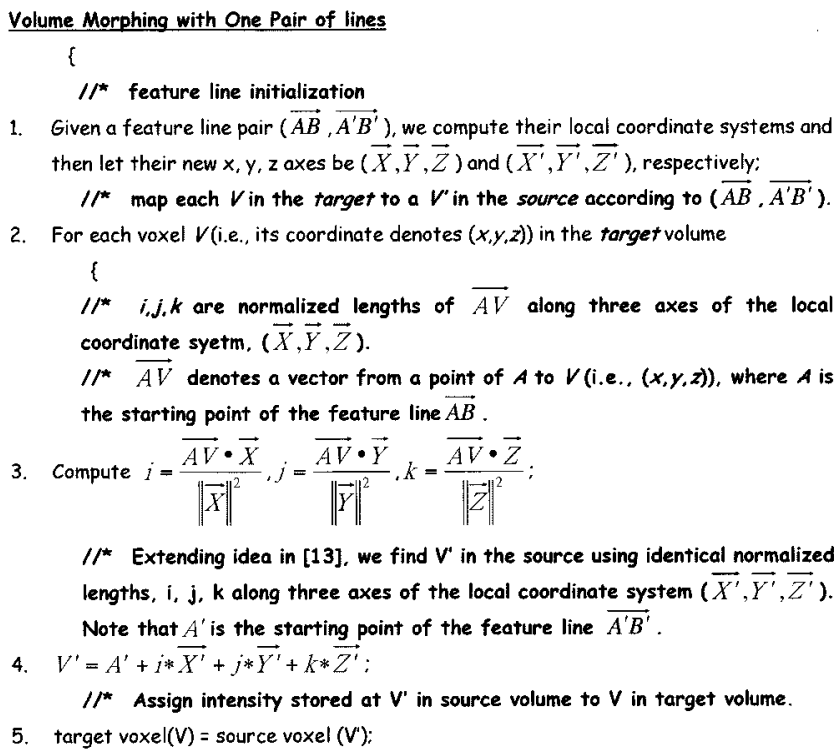

Fig. 4. Volume morphing algorithm using a single pair of feature lines.

target volume is initially assigned zero intensity. Using the 3-D volume morphing technique, we fill each voxel of the target volume data with the correct intensity. In this figure, we assume that there is a single pair of lines $\left(\overrightarrow{A B}, \overrightarrow{A^{\prime} B^{\prime}}\right)$ used to control volume morphing. Each feature line defined by two points ( $A, B)$ is represented as a vector $\vec{A} \vec{B}$ since this directed vector is used to define its local coordinate system. Whenever the user specifies each line $(A, B)$, the order of $(A, B)$ or $(B, A)$ must be carefully determined. This pair of corresponding lines in the source and target volume defines a coordinate mapping from the source voxel $V^{\prime}$ to the target voxel $V$. In the current implementation, we adopted reverse mapping to avoid the hole problem occurring in forward mapping from source to target [15]. The reverse mapping goes through the target volume voxel by voxel, and samples the correct voxel from the source volume data, as shown in Fig. 4.

Each feature line $\overrightarrow{A B}$ defines its local coordinate system using a $3-\mathrm{D}$ position $A$ as its origin and three mutually perpendicular vectors $(\vec{X}, \vec{Y}, \vec{Z})$, which define the directions of three coordinate axes. With regard to finding $(\vec{X}, \vec{Y}, \vec{Z})$, our method is described as follows. First, we let $\overrightarrow{A B}$ to be the $\vec{X}$ vector. Second, the axis $\vec{Y}$ is defined by $\overrightarrow{A B} \times \vec{x}$, where $\vec{x}$ is the $x$-axis of the volume data coordinate system and " $X$ " denotes the cross product of two vectors. If the value of $\vec{A} \vec{B} \times \vec{x}$ is a zero vector, we compute $\overrightarrow{A B} \times \vec{y}$ or $\vec{A} \vec{B} \times \vec{z}$ instead, where $\vec{y}$ and $\vec{z}$ are the $y$ and $z$-axis of the volume data coordinate system, respectively. Finally, we define the $\vec{Z}$ vector by the vector of $\vec{X} \times \vec{Y}$. Each voxel coordinate $V$ defined in the target volume coordinate system can be represented in terms of this new local coordinate system, i.e.,

$$
V=A+i * \vec{X}+j * \vec{Y}+k * \vec{Z}
$$

where

$$
i=\frac{\overrightarrow{A V} \bullet \vec{X}}{\|\vec{X}\|^{2}} \quad j=\frac{\overrightarrow{A V} \bullet \vec{Y}}{\|\vec{Y}\|^{2}} \quad k=\frac{\overrightarrow{A V} \bullet \vec{Z}}{\|\vec{Z}\|^{2}} .
$$

In (1), the terms $i, j, k$ are normalized lengths of $\vec{A} \vec{V}$ (i.e., a vector from $A$ to $V$ ) along directions of three coordinate axes $(\vec{X}, \vec{Y}, \vec{Z})$. For the corresponding feature line $\overrightarrow{A^{\prime} B^{\prime}}$ in the source volume data, we employ the same method to define its local coordinate system. Finally, a coordinate mapping from the target voxel $V$ to the source voxel $V^{\prime}$ is defined by

$$
V^{\prime}=A^{\prime}+i * \overrightarrow{X^{\prime}}+j * \overrightarrow{Y^{\prime}}+k * \overrightarrow{Z^{\prime}}
$$

where the local coordinate system of $\overrightarrow{A^{\prime} B^{\prime}}$ is defined by the origin $A^{\prime}$ and three mutually perpendicular vectors $\overline{X^{\prime}}, \overline{Y^{\prime}}, \overline{Z^{\prime}}$. Note that, in this mapping, both (1) and (2) use identical $i, j$, and $k$. We then assign the voxel intensity of $V^{\prime}$ in the source volume to that of $V$ in the target volume. Using the above approaches, we assign an intensity value to each voxel of the target volume data.

In the above pseudocode, a feature line pair $\left(\overrightarrow{A B}, \overrightarrow{A^{\prime} B^{\prime}}\right)$ is used to transform each voxel. In practical applications, we always specify multiple pairs of line segments to control volume morphing. Given $n$ pairs of feature lines, a weighted scheme is used to determine the voxel $V$ in the target volume that corresponds to the point $V^{\prime}$ of the source volume, i.e.,

$$
V^{\prime}=\sum_{i=1}^{n} V_{i}^{\prime} w_{i} / \sum_{i=1}^{n} w_{i} .
$$

The voxel $V_{i}^{\prime}$ is mapped from $V$ using a $i$ th feature line pair. Every $V_{i}^{\prime}$ is weighted by $w_{i}$ defined as $(d+\varepsilon)^{-2}$, where $d$ is the perpendicular distance from $V$ to the feature line $\overrightarrow{A B} ; \varepsilon$ is a small constant used to avoid division by zero. Like a single-line pair, we then assign an intensity stored at source voxel $\left(V^{\prime}\right)$ to a voxel $(V)$ of the target volume data. In this manner, we achieve multiple feature-lines volume morphing.

A performance drawback of the proposed volume morphing technique is that we need to compute the corresponding voxel in the source volume for each voxel in the target volume. To reduce this computation cost, we optimize the speed of warping by a piecewise linear approximation [17] described as follows. The target volume is considered as a big cube and it is virtually subdivided into several small sub-cubes. Each cube vertex and each sub-cube vertex is warped into the source volume. Using warped cube vertices, we also trilinearly interpolate the warped positions of sub-cube vertices. If the difference between the piecewise interpolated and actually warped results is within a range, we assume that voxels in the interior of a cube can be warped by trilinearly interpolating the warped positions of the cube vertices. In the reverse situation, we subdivide the cube into several small sub-cubes and we then recursively apply the above process to each sub-cube. For a $320 \times 320 \times 151 \mathrm{CT}$ 


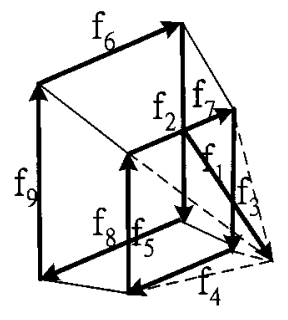

(a)

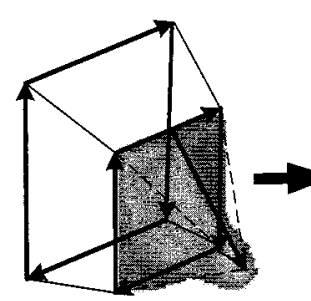

(b)

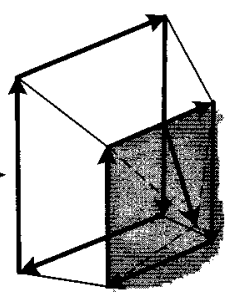

(c)

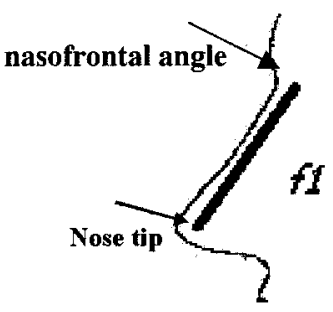

(d)

Fig. 5. Organization of two sets of feature lines.

head data with nine feature lines, the above optimization significantly reduces morphing time from several minutes to less than 1 min on the Intel Pentium II 233-MHz PC platforms with 128-M memory. To interactively reshape nose using 3-D morphing, we always scale this $320 \times 320 \times 151 \mathrm{CT}$ head data by 0.5 in three axes. With this reduction, the executing time of morphing is about $10 \mathrm{~s}$ on the same platform. This performance is sufficient for surgeons to interactively simulate nose surgery. In Section IV, we will show exact morphing time and also examine the corresponding measurement-accuracy degradation in postoperative results due to this volume reduction.

We next give detail as to how the features of the nose are specified. As mentioned earlier, surgeons need to specify two sets of line features: one is for the preoperative and the other is for the postoperative volume data. In the proposed system, each set consists of nine feature lines, as shown in Fig. 5(a). Surgeons define these features by clicking mouse buttons on the object surface. Among these nine lines, feature lines $\left\langle f_{2}, f_{3}, \ldots, f_{9}\right\rangle$ are identical for both source and target volume. The main reason for this design is that the feature-based morphing method globally influences the warping of each voxel. However, the purpose of our system is only to reshape the nose rather than other portions of the head. Therefore, we use identical lines $\left\langle f_{2}, f_{3}, \ldots, f_{9}\right\rangle$ to make the major portions of head almost unchanged [i.e., target voxel $(V)=$ source voxel $\left(V^{\prime}\right)$ ], except the nose area. In our design, only the feature line $f_{1}$ contributes to the change on the nose. Usually, in our practice, by means of the interface like Fig. 2, we determine line feature $f_{1}$, as in Fig. 5(d), where one point is initially located at the nose tip and the other point is near the nasofrontal angle. Surgeons arbitrarily adjust $f_{1}$ 's positions according to the patient's desire to perform simulation. For example, in Fig. 5(b) and (c), we adjust one end point (i.e., for the nose tip) of the feature line $f_{1}$ and we can easily obtain two different sizes of the nose. We will next give more detail as to how the other eight features $\left\langle f_{2}, f_{3}, \ldots, f_{9}\right\rangle$ are specified.

In human anatomy, there are three imaginary planes used to describe the pose of body parts, which are described as follows.

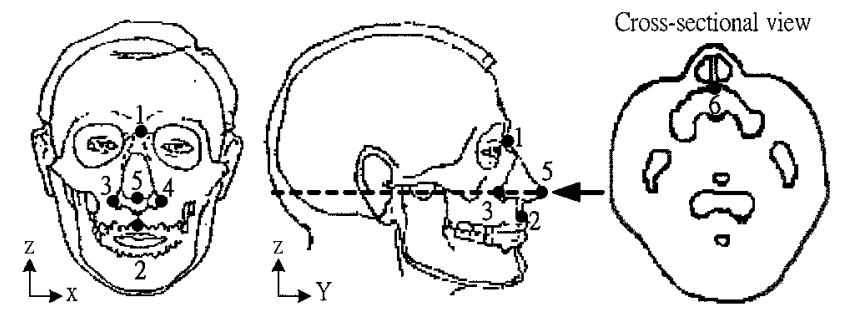

Fig. 6. Six feature points in the head structure. (1) Nasal bones at the top of nose. (2) White line of the lip. (3) Alare (left): the lateral point on the flare or wing of the nose. (4) Alare (right). (5) Tip of the nose. (6) Maxilla.

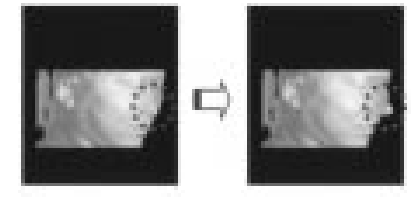

(a)

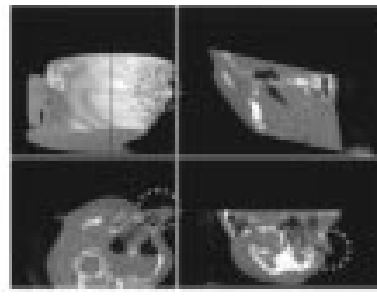

(b)
Fig. 7. Example of the surgical simulation using the proposed system. (a) The change in nose after morphing. (left-hand side) Preoperative nose. (right-hand side) Postoperative nose. (b) Postsurgical 3-D rendered results and three cross-sectional images.

- Median plane [parallel to the $Y Z$-plane in Fig. 6 (lateral view of the head)]: this vertical plane cuts through the center of the body, dividing the body into equal right- and left-hand-side halves.

- Coronal plane [parallel to the $X Z$-plane in Fig. 6 (frontal view of the head)]: this vertical plane divides the body into front and back halves.

- Transverse horizontal planes (parallel to the $X Y$-plane in Fig. 6 (cross-sectional view of the head): these planes are at right angles to both the median and coronal planes.

Eight features $\left\langle f_{2}, f_{3}, \ldots, f_{9}\right\rangle$ define six planes of a rectangular bounding box as follows. For this purpose, on the frontal view of the head, surgeons need to specify several feature points labeled and explained in Fig. 6. First, two vertical planes cutting through the $Z$-axis and feature points 1 and 2, respectively, are defined. Second, two vertical planes cutting through the $X$-axis and feature points 3 and 4 , respectively, are defined. Next, surgeons first locate a maxilla point (feature point 6 ) from a cross-section view (parallel to the $X Y$-plane). One vertical plane then cutting through the $Y$-axis and feature point 6 is defined. This plane is called the "based plane" and is later used to measure the nasal structure. Finally, as illustrated in Fig. 6, we define another plane parallel to the based plane and at a distance $d$ (i.e., usually about 20-30 pixel wide) from the based plane. Now, we have already defined six planes for a rectangular bounding box. Therefore, two end points of each feature line $\left\langle f_{2}, f_{3}, \ldots, f_{9}\right\rangle$ can be easily found. In Fig. 6 , the feature point 5 (the tip of the nose) is used to initially define one end point of the feature line $f_{1}$, as mentioned earlier.

Fig. 7 shows an example of preoperative and postoperative results. Both surgeons and patients preview 3-D postoperative results and compare visual difference with those of preoperative data. In this example, we would like to increase the pointedness 


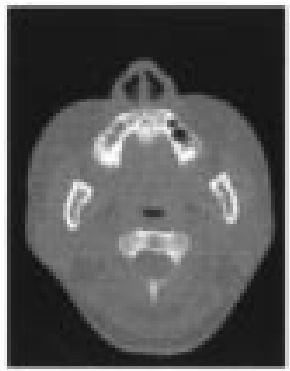

(a)

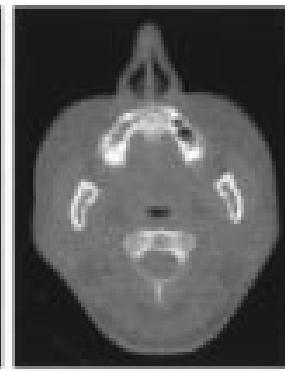

(b)

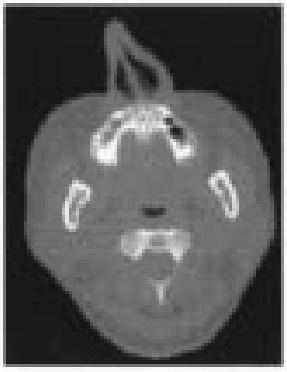

(c)

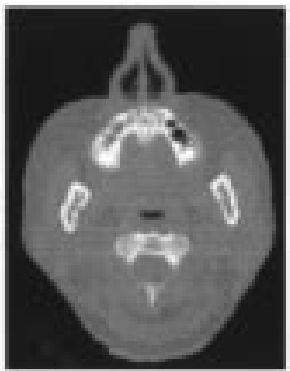

(d)

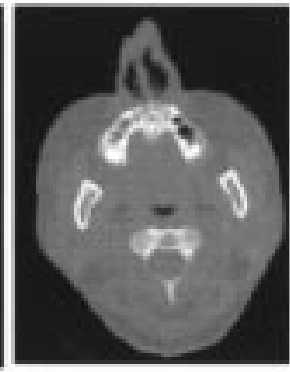

(e)

Fig. 8. Comparison between a line and point feature for $f_{1}$. (a) Original nose shape. (b) Also augmented by the proposed method. (c) Pose of the nose is slightly altered. (d) and (e) Results with attempt to return the original pose of nose by using a line feature (d) and a point (e) for $f_{1}$, respectively.

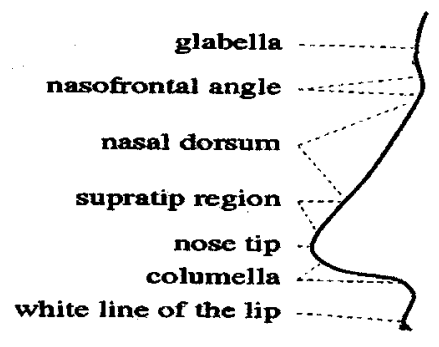

Fig. 9. Human nose with its regions and landmarks.

of the nose tip. It is very intuitive to place both features $f_{1}$ and $f_{1}^{\prime}$ like Fig. 5(b) and (c) to achieve this effect.

In our previous study [12], we proposed a surgical operation called augmentation operator that used "point feature" instead of "line segment" for $f_{1}$. The purpose of this slight modification is that the "line feature" allows better control of the reshape of the whole nose than point features. To verify this, we perform the following experiment. In Fig. 8, we first lengthen the shape of the nose from (a) to (b). Next, we slightly change the pose of the nose, as shown in (c). Now, we attempt to adjust the pose back to the original one in (b) by using a line feature in (d) and a point feature in (e), respectively, for $f_{1}$. Based on results obtained from (d) and (e), the line feature of $f_{1}$ performs better than "point feature" to control the reshape of the nose.

\section{EXPERIMENTAL RESUlTS AND QUANTITATIVE MEASUREMENT}

After surgical simulation, surgeons can view both 3-D postoperative results and three cross-sectional images to examine the validity of this simulation. To judge the success of this simulation, the quantitative measurement of parameters describing the nasal structure are crucial. In Fig. 9, we show the profile of a nose with the medical terminology in rhinoplasty. Achermann et al. [1] measured related nasal parameters based on the pictures of the profiles of the patients. However, all measurements were limited to the 2-D measurement or the parameters measured from a 2-D photograph. The parameters that can be better measured in a 3-D way, such as volume change in nasal structure, are still lacking, though they are crucial for surgical planning. In contrast, the proposed system measures most nasal parameters in 3-D. We should mention we are not the first to use 3-D measures such as volume; surface subtraction as quantitative measures were already introduced in the cadaver study of

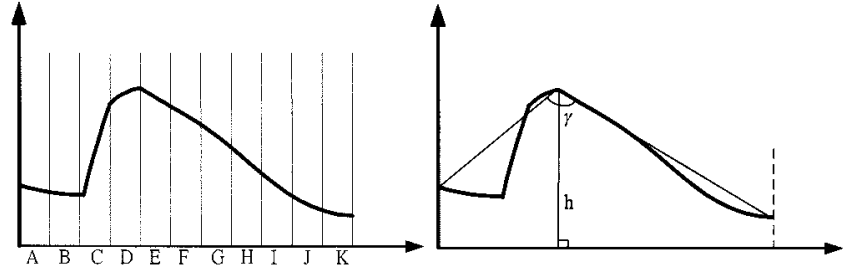

Fig. 10. Nasal parameters.

facial surgery simulation [2]. These include most of the parameters in our paper, except a position of the nose tip and a position difference of the nose tip.

Similar to [15], the nasal profile is divided into 11 parts of equal width $(A-K)$ [as shown in Fig. 10(a)]. The nose tip has been manually selected as feature point 5 (termed $P_{5}$ ) in Fig. 6 to define the feature line $f_{1}$ in Section II. In Fig. 10(b), two feature points (the nasal bones) at the top of the nose (feature point 1, i.e., $P_{1}$, in Fig. 6) and the white line of the lip (feature point 2, i.e., $P_{2}$, in Fig. 6) with the nose tip form a triangle for which the angle (unit: degree) $\gamma$ (i.e., $\angle P_{2} P_{5} P_{1}$ ) is measurement of the pointedness of the nose tip. In Section III, we define a based plane cutting through the $Y$-axis and feature point 6 in Fig. 6. In Fig. 10(b), the height $(h)$ of the nose is defined as the perpendicular distance from the nose tip to the based plane. Similarly, the volume measurements in Fig. 10(a) from $A$ to $K$ are also in reference to this based plane (i.e., to measure volume above this plane). Recall in Section III, that feature points 1 and 2 define two vertical planes cutting through the $Z$-axis. These two vertical planes with the based plane define a region of measurement. We simply vertically cut this region (through the based plane) into 11 parts of equal width to form subregions $A$ to $K$.

An example of nose surgery simulated in the proposed system is shown in Fig. 11. In this figure, from top to bottom, we show one preoperative (top) and the other two postoperative (middle and bottom) 3-D head surface. To show a better visual difference among these three results, the cross-sectional view of nose and nose volume are extracted and independently shown in the middle of this figure. Furthermore, on the right-hand side, we show 3-D measurement of nasal profile [measurement unit is millimeter $(\mathrm{mm})$ ] before and after the simulation. These figures can assist surgeons in comparing quantitative differences among preoperative and postoperative nasal changes in size. Our simulations are explained as follows. The pose of nose in Fig. 11(a) is not very straight. Thus, during the first surgery, we attempt 


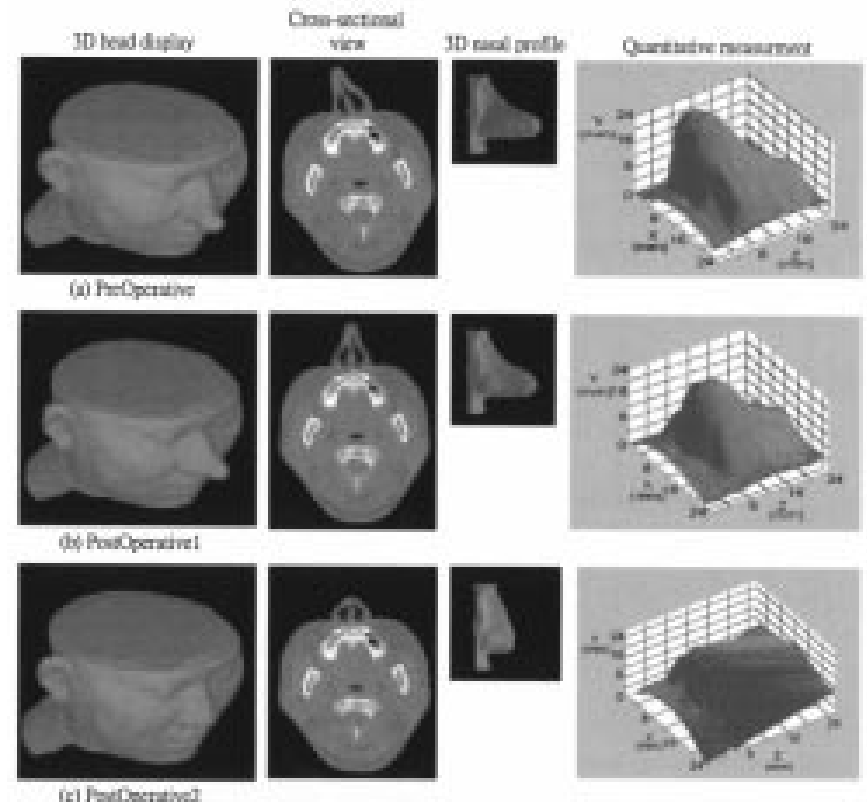

Fig. 11. Comparisons among preoperative and two postoperative results. On the right-hand side, we visualize and measure the nasal profile in 3-D. The measurement unit is millimeters.

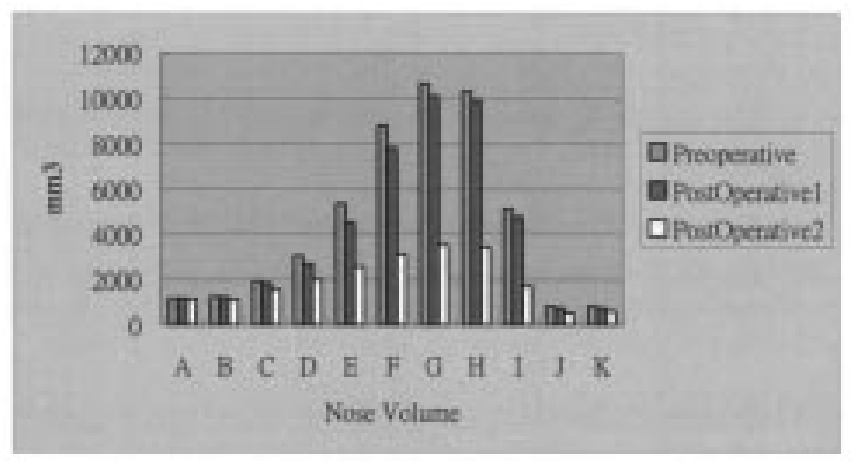

Fig. 12. Volume measurement of nasal profile for each region.

to adjust the pose of the nose, as shown in Fig. 11(b). However, the patient still feels the size of the nose is too big. In Fig. 11(c), we attempt to make the nose smaller to meet the requirement of the patient. Both surgical simulations are accomplished by simply changing the positions of the feature line $f_{1}$. Next, we tabulate the simulated results in Fig. 12 and Table I. In this table, we can easily understand quantitative difference of 3-D nasal profile among preoperative and postoperative cases. Surgeons use this table to know the volume change (in $\mathrm{mm}^{3}$.) of a nose shape in each region. For the postoperative 1, the nose pose is adjusted in comparison with the preoperative one. In Table I, two parameters, i.e., nose-tip and vector $\boldsymbol{V}$, show these evidences. These two parameters are measured in the CT volume coordinate space. The position of the nose tip (i.e., Nose-tip) was moved from $(129,33,61)$ to $(149,33,61)$ in voxel space and $V$ denotes the pose of this nose is altered in CT voxel space. Additionally, in this operation, the volume size slightly decreases in regions $\mathrm{C}$-I, whereas the other regions remain nearly constant (see Fig. 12). Therefore, the nose does not change too much in size. On the other hand, postoperative 2 results show the nose
TABLE I

QuANTITATIVE MEASUREMENT OF NASAL PARAMETERS, WHERE MEASUREMENT UNIT FOR REGIONS $A-K$ IS $\mathrm{mm}^{3}$, FOR ANGLE $\gamma$ IS DegreE, AND FOR HeIGHT $h$ Is MiLlimeters. THE POSITION OF THE NOSETIP AND THE MOVEMENT Vector $V$ IS MEASURED IN THE ORIGINAL CT VOlume VoXel COORDinate SyStem

\begin{tabular}{|c|c|c|c|}
\hline & PreOperational & PostOperative1 & PostOperative2 \\
\hline Volume $\mathrm{A}\left(\mathrm{mm}^{3}\right)$ & 1168 & 1168 & 1121 \\
\hline Volume B $\left(\mathrm{mm}^{3}\right)$ & 1242 & 1227 & 1128 \\
\hline Volume $\mathrm{C}\left(\mathrm{mm}^{3}\right)$ & 1947 & 1842 & 1566 \\
\hline Volume D $\left(\mathrm{mm}^{3}\right)$ & 3058 & 2687 & 1995 \\
\hline Volume $\mathrm{E}\left(\mathrm{mm}^{3}\right)$ & 5374 & 4525 & 2567 \\
\hline Volume $\mathrm{F}\left(\mathrm{mm}^{3}\right)$ & 8823 & 7837 & 3144 \\
\hline Volume $\mathrm{G}\left(\mathrm{mm}^{3}\right)$ & 10651 & 10112 & 3535 \\
\hline Volume $\mathrm{H}\left(\mathrm{mm}^{3}\right)$ & 10321 & 9930 & 3436 \\
\hline Volume I $\left(\mathrm{mm}^{3}\right)$ & 5109 & 4773 & 1731 \\
\hline Volume $\mathrm{J}\left(\mathrm{mm}^{3}\right)$ & 826 & 760 & 523 \\
\hline Volume $\mathrm{K}\left(\mathrm{mm}^{3}\right)$ & 820 & 759 & 606 \\
\hline Angle $\gamma$ (degree) & 70.95 & 72.42 & 110.54 \\
\hline $\begin{array}{ll}\mathrm{H} & (\mathrm{mm}) \\
\end{array}$ & 22.37 & 22.37 & 13.53 \\
\hline NoseTip & $(129,33,61)$ & $(149,33,61)$ & $(149,53,61)$ \\
\hline & PreOperative $\rightarrow$ PostOperative 1 & \multicolumn{2}{|c|}{ PostOperative1 $\rightarrow$ PostOperative 2} \\
\hline $\boldsymbol{V}$ & $(20,0,0)$ & \multicolumn{2}{|r|}{$(0,20,0)$} \\
\hline
\end{tabular}

becomes much smaller and its tip becomes less pointed (i.e., $\gamma$ becomes larger) than those of both preoperative and postoperative 1 . These changes can be observed from the changes in regions $A-K$ in Fig. 12. Table I and Fig. 12 provide very valuable information to surgeons. With this information, surgeons can plan operations and know where to surgically and modify how much change is required for each region. This table also provides additional information about the pointedness of the nose tip such as angle $\gamma$ and height $h$. For example, height $h$ (measurement unit: millimeters) is reduced from postoperative $1(41 \mathrm{~mm})$ to postoperative $2(22 \mathrm{~mm})$. Similarly, the parameter $V(0,20,0)$, indicates the nose tip moves toward the based plane (i.e., less pointed) from the postoperative 1 to 2 . Additionally, the nose of postoperative 2 becomes less pointed (i.e., $\gamma$ becomes larger) than those of both preoperative and postoperative 1 . These parameters of a nose tip provide objective observation and measurement to both patients and surgeons. These results also provide evidences that both simulated surgeries improve the pointedness of the nose tip.

In Section III, to interactively reshape the nose using 3-D morphing, we always scale CT head data by 0.5 in three axes. After morphing, we will apply Gaussian filter to scale each dimension back to the original size. We then conduct the same measurements as shown in Table I. In Table II, we list our measurements of volume sizes from $A$ to $K$ and error ratio in comparison with those of experiments on nonscaled data. Experimental results show that the measurement-accuracy degradation due to this scale down is insignificant. Next, in Table III, we also show the timing for the various operations for a cycle of surgical simulation. Our computing platform is the Intel Pentium II 233-MHz PC with 128-M memory. In this table, we know the major cost in this cycle is spent on volume morphing. Finally, we show various volume morphing timings about optimized (i.e., by $3-\mathrm{D}$ piecewise linear interpolation) versus nonoptimized and 
TABLE II

VOLUME MEASUREMENT USING SCALED CT DATA. $R 1$ AND $R 2$ IS THE ERROR RATIO FOR POSTOPERATIVE 1 AND 2 IN COMPARISON WITH THOSE (FROM $A$ to $K$ ) Of Nonscales Volume Data

\begin{tabular}{l|c|c|c|l}
\hline & $\begin{array}{l}\text { Post- } \\
\text { Operative1 }\end{array}$ & $\begin{array}{l}\text { Post- } \\
\text { Operative2 }\end{array}$ & $\begin{array}{l}\text { R1 } \\
(\%)\end{array}$ & $\begin{array}{l}\text { R2 } \\
(\%)\end{array}$ \\
\hline Volume $\mathrm{A}\left(\mathrm{mm}^{3}\right)$ & 1149 & 1103 & 1.69 & 1.60 \\
\hline Volume B $\left(\mathrm{mm}^{3}\right)$ & 1215 & 1113 & 1.06 & 1.32 \\
\hline Volume $\mathrm{C}\left(\mathrm{mm}^{3}\right)$ & 1831 & 1550 & 0.70 & 1.02 \\
\hline Volume D $\left(\mathrm{mm}^{3}\right)$ & 2675 & 1979 & 0.60 & 0.80 \\
\hline Volume $\mathrm{E}\left(\mathrm{mm}^{3}\right)$ & 4512 & 2550 & 0.50 & 0.66 \\
\hline Volume $\mathrm{F}\left(\mathrm{mm}^{3}\right)$ & 7826 & 3130 & 0.34 & 0.44 \\
\hline Volume $\mathrm{G}\left(\mathrm{mm}^{3}\right)$ & 10098 & 3517 & 0.39 & 0.50 \\
\hline Volume $\mathrm{H}\left(\mathrm{mm}^{3}\right)$ & 9914 & 3420 & 0.46 & 0.46 \\
\hline Volume I $\left(\mathrm{mm}^{3}\right)$ & 4761 & 1715 & 0.69 & 0.92 \\
\hline Volume J $\left(\mathrm{mm}^{3}\right)$ & 749 & 509 & 2.10 & 2.67 \\
\hline Volume $\mathrm{K}\left(\mathrm{mm}^{3}\right)$ & 748 & 591 & 1.81 & 2.47 \\
\hline
\end{tabular}

TABLE III

Timing For the VARIOUS OPERATIONS IN A CyCLE OF SURGiCAl SimUlation

\begin{tabular}{c|c|c}
\hline & $\begin{array}{c}\text { PreOperative } \rightarrow \\
\text { PostOperative1 }\end{array}$ & $\begin{array}{c}\text { PostOperative } 1 \rightarrow \\
\text { PostOperative } 2\end{array}$ \\
\hline $\begin{array}{c}\text { Scale }\left(320^{*} 320^{*} 151\right) \rightarrow \\
\left(160^{*} 160^{*} 75\right)\end{array}$ & 1.52 seconds & 1.52 seconds \\
\hline Volume Morphing & 9.94 seconds & 11.82 seconds \\
\hline Creating SB structure & 2.75 seconds & 2.72 seconds \\
\hline $\begin{array}{c}\text { SB Rendering } \\
\text { Scale }\left(160^{*} 160^{*} 75\right) \rightarrow \\
\left(320^{*} 320^{*} 151\right)\end{array}$ & 0.11 seconds & 0.11 seconds \\
\hline Measurements & 1.53 seconds & 1.53 seconds \\
\hline
\end{tabular}

TABLE IV

Timing COMPARISONS FOR DIFFERENT KINDS OF MORPHING CONFIGURATIONS IN FIG. 11

\begin{tabular}{c|c|c}
\hline & Optimized & Non-optimized \\
\hline $\begin{array}{c}\text { Preoperative } \rightarrow \text { Postoperative I }\left(160^{*} 160^{*} 75\right) \\
\text { Postoperative } 1 \rightarrow \text { Postoperative } 2 \\
\left(160^{*} 160^{*} 75\right)\end{array}$ & 9.94 seconds & 204.21 seconds \\
\hline $\begin{array}{c}\text { Preoperative } \rightarrow \text { Postoperative } 1\left(320^{*} 320^{*} 151\right) \\
\text { Postoperative } 1 \rightarrow \text { Postoperative } 2 \\
\left(320^{*} 320^{*} 151\right)\end{array}$ & 119.21 seconds & 169.07 seconds \\
\hline
\end{tabular}

scale down versus nonscaled in Table IV. This table can help us understand the importance of optimization and scale down in the current implementation to achieve a reasonably interactive speed.

\section{CONCLUSION}

The human face plays a key role in interpersonal relationships. Prediction of postsurgical morphology and appearance of human faces for patients with facial deformities is a critical issue in facial surgery. Surgeons face the problem of designing repair plans or compensation procedures before an actual surgery is carried out. Rhinoplasty, or surgery to reshape the nose, is one of the most common of all facial surgeries. In this paper, we introduce a system to assist surgeons in planning rhinoplasty. This system integrates image processing and computer graphics techniques. To understand the different spatial relationship of the nose and face, skin and bone structure, etc., this system provides interactive 3-D SB rendering and image segmentation tools. Surgeons preview 3-D models as well as three cross-sectional images to examine their relationships. In this manner, it has the potential to find the best procedures to perform surgery. The proposed system exploits the new idea to apply morphing technique for rhinoplasty. Using morphing feature lines, surgeons can freely adjust a change of the nose structure. At the same time, patients can preview the postsurgical change in their noses and give their feedback about their personal feelings regarding this change. Surgeons can then further reshape the nose to best satisfy the desire of the patients. Our system also provides quantitative measurements, including features of the nose such as the nose tip position. It also measures nose volume for presurgical and postsurgical simulation. This information helps the surgeons in designing appropriate implants to augment the nose. We believe this system has the potential to improve the quality of rhinoplasty and to provide an informative communication interface between surgeons and patients.

\section{ACKNOWLEDGMENT}

The authors would like to present their sincere thanks to Prof. Y.-N. Sun, Department of Computer Science and Information Engineering, National Cheng-Kung University, Tainan, Taiwan, R.O.C., and Dr. S.-C. Lin, National Cheng-Kung University Hospital, Tainan, Taiwan, R.O.C., for their valuable discussions, medical expertise, and medical CT volume data. The authors also extend special thanks to Y.-C. Lin, Visual System Laboratory, National Cheng-Kung University, for his programming efforts in the early stage of system development. The authors also gratefully acknowledge the anonymous reviewers, whose helpful comments improved the quality of this paper.

\section{REFERENCES}

[1] B. Achermann, R. Peleg, J. Xiaoyi, H. Bunke, D. Feinendegen, Y. Bruhlmann, and H. Tschopp, "Computed-based assistance of surgeons in the judgement of plastic nose surgery (rhinoplasty)," in IEEE Int. Adv. Intell. Mechatron. Conf., 1997, pp. 21-25.

[2] V. V. Patel, M. W. Vannier, J. L. Marsh, and L.-J. Lo, "Assessing craniofacial surgical simulation," IEEE Comput. Graph. Appl., pp. 46-54, Jan. 1996.

[3] L. J. Lo, "Craniofacial computer assisted surgical planning and simulation," Clin. Plast. Surgery, vol. 21, no. 4, pp. 501-516, Oct. 1994.

[4] M. W. Vannier, J. L. Marsh, and J. O. Warren, "Three-dimensional CT reconstruction images for craniofacial surgical planning and evaluation," Radiology, vol. 150, no. 1, pp. 179-185, Jan. 1984.

[5] A. D. Linney, J. P. Moss, R. Richards, C. A. Mosse, S. R. Grindrod, and A. M. Coombes, "Use of 3D visualization system in the planning and evaluation of facial surgery," in Proc. SPIE Biostereometr. Applicat. Conf., Boston, MA, 1990, pp. 1-10.

[6] R. M. Koch, M. H. Gross, F. R. Carls, D. F. von Buren, G. Fankhauser, and Y. I. H. Parish, "Simulating facial surgery using finite element models," in Proc. ACM SIGGRAPH'96 Annu. Conf., pp. 421-428.

[7] K. Waters, "A muscle model for animating three-dimensional facial expression," in Proc. ACM SIGGRAPH'87 Annu. Conf., pp. 17-24.

[8] Y. Lee, D. Terzopoulos, and K. Waters, "Realistic face modeling for animation," in Proc. ACM SIGGRAPH'95 Annu. Conf., pp. 55-62.

[9] W. A. Aulsebrook, M. Y. Iscan, J. H. Slabbert, and P. Becker, "Superimposition and reconstruction in forensic facial identification-A survey," Forens. Sci. Int., vol. 75, no. 2-3, pp. 101-20, 1995.

[10] S. Miyasaka, M. Yoshino, K. Imaizumi, and S. Seta, "The computeraided facial reconstruction system," Forens. Sci. Int., vol. 74, no. 1-2, pp. 155-65, 1995.

[11] D. H. Ubelaker and G. O' Donnell, "Computer assisted facial reconstruction,” J. Forens. Sci., vol. 37, pp. 155-62, 1992. 
[12] T.-Y. Lee, Y. N. Sun, Y. C. Lin, L. Lin, and C. N. Lee, "Three-dimensional facial reconstruction and plastic surgical simulation," IEEE Trans. Inform. Technol. Biomed., vol. 3, pp. 214-220, Sept. 1999.

[13] J. K. Udupa and D. Odhner, "Fast visualization, manipulation, and analysis of binary volumetric objects," IEEE Comput. Graph. Appl., pp. 53-62, Nov. 1991.

[14] T.-Y. Lee, W. L. Weng, C. H. Lin, and Y. N. Sun, "Interactive voxel surface rendering in medical applications," Comput. Med. Imaging Graph., vol. 23, no. 4, pp. 193-200, July-Aug. 1999.

[15] T. Beier and S. Neely, "Feature-based image metamorphosis," in Proc. SIGGRAPH'92, pp. 35-42.

[16] J. K. Udupa, D. Odhner, S. Samarasekera, R. Goncalves, K. Iyer, P. Vengupal, and S. Furuie, "3DVIEWNIX: An open, transportable, multidimensional, multi-modality, multi-parametric imaging software system," Proc. SPIE, vol. 2164, pp. 58-73, 1994.

[17] A. Lerios, C. D. Garfinkle, and M. Levoy, "Feature-based volume metamporphosis," in Proc. SIGGRAPH'95, pp. 449-464.

Tong-Yee Lee was born in Tainan, Taiwan, R.O.C., in 1966. He received the B.S. degree in computer engineering from the Tatung Institute of Technology, Taipei, Taiwan, R.O.C., in 1988, the M.S. degree in computer engineering from the National Taiwan University, Taiwan, R.O.C., in 1990, and the Ph.D. degree in computer engineering from Washington State University (WSU), Pullman, in 1995.

$\mathrm{He}$ is currently an Associate Professor in the Department of Computer Science and Information Engineering, National Cheng-Kung University, Tainan, Taiwan, R.O.C. During the summer of 1996, he was a Visiting Research Professor with the School of Electrical Engineering and Computer Science, WSU. Since 1992, he has been involved with parallel rendering and computer graphics. He has authored or co-authored over 70 technical papers in referred journals and conferences. His current research interests include image-based rendering, computer graphics, visualization, virtual reality, surgical simulation, distributed and collaborative virtual environment, parallel processing, and heterogeneous computing.

Dr. Lee was a guest associate editor for the IEEE TRANSACTIONS ON INFORMATION TECHNOLOGY IN BIOMEDICINE in 2000 and 2001.
Chao-Hung Lin was born in Koushung, Taiwan, R.O.C., in 1973. He received the B.S. degree in computer science/engineering from Fu-Jen University, Taiwan, R.O.C., in 1997, the M.S. degree in computer engineering from the National Cheng-Kung University, Tainan, Taiwan, R.O.C., in 1998, and is currently working toward the Ph.D. degree in computer science and information engineering at the National Cheng-Kung University.

His research interests include computer graphics, image processing, virtual reality, visualization, and interactive rendering.

Han-Ying Lin was born in Tainan, Taiwan, R.O.C., in 1977. He received the B.S. degree in computer science/engineering from Feng-Chia University, Taichung, R.O.C., in 1999, and is currently working toward the M.S. degree in computer science and information engineering at the National Cheng-Kung University, Tainan, Taiwan, R.O.C.

His research interests include computer graphics, image processing, 3-D volume and shape morphing, virtual reality, and visualization. 